\title{
Complexity Analysis of Overall Behavior of Network Based on Topology Structure
}

\author{
Liping Zhang \\ Sichuan Information Technical College, China \\ 55217096@qq.com
}

Keywords: topology network; fluctuation complexity; complexity analysis

\begin{abstract}
In this paper, the complexity of the network integrity is analyzed based on the topology structure. The generalized scale free network model is used as the research object, which is defined to describe the complex degree of the overall behavior of the network. In order to understand the influence of network topology on the overall behavior of the computer network, the influence of topology structure on the overall behavior of the network is analyzed by using the fluctuation complexity of the traffic flow.
\end{abstract}

\section{Introduction}

It is proved that the phenomenon of phase transformation is often occurred during the evolution of complex systems. Phase is the state of the system. And phase change, simply said, is the dynamical properties of the system from disorder to order, or from order to disorder state. Therefore, in statistical physics, phase transformation is when the control parameter of the system is lower than a certain critical value, the system is in a stable state, and once crossed the critical value, the dynamic properties of the system will be transferred to another state.

However, the foundation of this work and its conclusions are based on the uniform topology structure. In the network model, the assumption is that the network node degree distribution is uniform, and the influence of the heterogeneity of the network topology on the overall behavior of the network is ignored. Also, the correlation of the behavior of each individual node is absent during the evolution course.

In order to understand the influence of network topology on the overall behavior of the computer network, this paper analyzes the overall behavior of the network system based on the concept of the critical phase transition in the generalized network model.

\section{Generalized Scale Free Network Model}

The scale free network with initial attraction is a class of generalized scale free networks proposed by Dorogovtsev et al. It is an extension of the BA network model, and its construction algorithm is similar to the BA model which includes two parts, the growth and preference characteristic.

(1) The growth characteristic: Starting from the initial m0 node, in each time step, it will add a new node. Then m nodes are randomly selected from all nodes in the network, and the new nodes are connected with each of these nodes.

(2) The preference characteristic: New joined node will chose an old point i to connect and the connection probability of new point and old point is pi. The relationship between pi and I is shown as follows.

$$
p_{i}=\frac{A_{i}}{\sum_{j} A_{j}}=\frac{\alpha+k_{i}}{\sum_{j}\left(\alpha+k_{i}\right)}
$$

Where ki is the node degree of node $\mathrm{i} ; \alpha$ is the initial attraction degree for the node which means the initial ability to capture the new node; after t time steps, the number of nodes will be $N=m_{0}+t$ and the number of edges will be $m t$. 
Compared with the BA model, the model of the scale free network introduces the initial attraction. The scale characteristics of the network topology obtained by the above construction method will be smoothly controlled by the initial attraction degree. The probability distribution of the degree of network nodes can be expressed as follows.

$$
P(k) \propto k^{-\gamma}
$$

Where $\gamma$ is a function about initial attraction $\alpha$. The relationship can be expressed as,

$$
\gamma=3+\alpha / m ; \quad \alpha \in[-m, \infty)
$$

With Figure 1, the control process of $\gamma$ is presented. The graph describes the network node degree of variance s and the network of the average node degree $\mathrm{K}$ along with the change of $\gamma$.

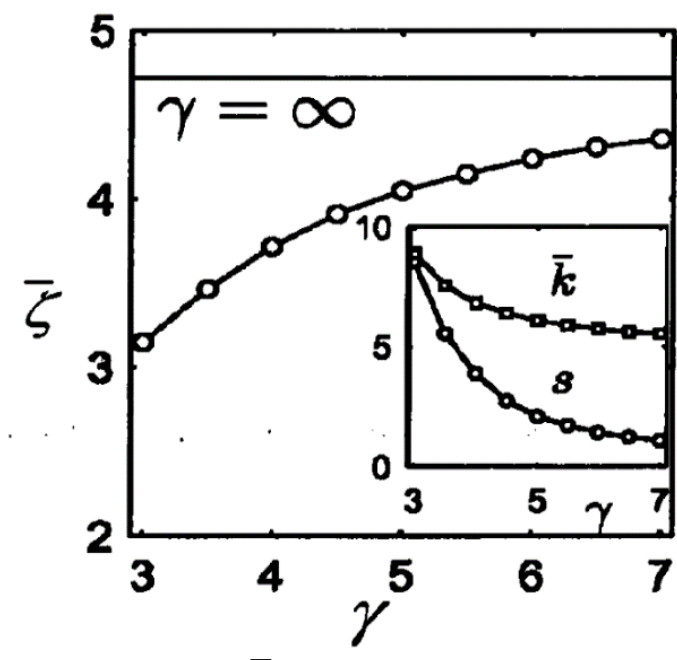

Fig 1. $\bar{\zeta}$ and $\bar{k}$ vs $\gamma$ on free-scale network

\section{Fluctuation Complexity of Network Traffic}

Now, according to the general definition of the complexity of the Bates fluctuation, the definition of fluctuation complexity of network traffic is presented by combining the characteristics of the evolution model of the network behavior.

Next, we will define the fluctuation complexity of the network traffic based on the node pair state space $S_{\text {pair }}$.

The node in the network is set as $(\mathrm{x}, \mathrm{y})$. The probability of the node in $S_{i}=\left(s_{i 1}, s_{i 2}\right)$ state is $p_{i}$. The equation can be expressed as

$$
p_{i}=p\left(S_{i}\right)=p\left(s_{i 1}, s_{i 2}\right)=p s_{i 1} \bullet p\left(s_{i 2} \mid p s_{i 1}\right)=p s_{i 2} \bullet p\left(s_{i 1} \mid p s_{i 2}\right)
$$

Where $p_{i}$ is the probability when the node $\mathrm{x}$ is in the state of $s_{i 1}$ and the node $\mathrm{y}$ is in the state of $s_{i 2}$.

Here, $p_{i j}$ is used to present the probability when the node state is changed from $S_{i}$ to $S_{j}$. And $p_{i \rightarrow j}$ is used to present the transition probability of the state change. $p_{i \rightarrow j}$ satisfies the equation as follows

$$
\begin{gathered}
\sum_{j=1}^{L^{2}} p_{i \rightarrow j}=1 \\
p_{i j}=p_{i} p_{i \rightarrow j}=p_{j \rightarrow i} p_{j}
\end{gathered}
$$

$I_{i j}^{+}=-\log p_{i \rightarrow j}$ is used to describe the information quantity during the state change. $I_{i j}^{-}=-\log p_{i \leftarrow j}$ is used to describe the information loss during the state change. 
$\overline{I^{+}}=-\sum_{i} \sum_{j} p_{i j} \bullet \log p_{i \rightarrow j} \quad$ is used to describe the average value of state transition information gain.

The average information loss can be describe as $\overline{I^{-}}=-\sum_{i=1}^{L} \sum_{i=1}^{L} p_{i j} \cdot \log p_{i \leftarrow j}$

Through these variables, the average net information can be obtained.

$$
\bar{\Delta} \bar{I}=-\sum_{i}^{L} p_{i} \log p_{i} \sum_{j}^{L} p_{i \rightarrow j}-\sum_{j}^{L} p_{j} \log p_{j} \log p_{j} \sum_{i}^{L} p_{j \rightarrow i}=0
$$

According to the definition of Bates fluctuation complexity, the fluctuation complexity of node $(\mathrm{x}, \mathrm{y}) C_{f}$ and the average fluctuation complexity $\overline{C_{f}}$ can be described as

$$
\begin{gathered}
C_{f}=\overline{[\Delta I]^{2}}-[\overline{\Delta I}]^{2}=\overline{[\Delta I]^{2}}=\sum_{i}^{L} \sum_{j}^{L} P_{i j}\left[\log \frac{p_{i}}{p_{j}}\right]^{2} \\
\overline{C_{f}}=\frac{1}{M(M-1)} \sum_{x \in V} \sum_{y \in V} C_{f}
\end{gathered}
$$

\section{Conclusion}

The Relationship between Network Topology Structure and the Complexity of Network Behavior. The influence of topology structure on the overall behavior of the network is analyzed by using the fluctuation complexity of the traffic flow. With the increase of $\gamma$, the heterogeneity of the network topology is reduced, so that the distribution of the traffic in each node is more uniform, and the degree of synchronization of the network is improved. Therefore, under a certain flow rate input rate, the state of each node will become more consistent, most of the state is concentrated in the neighborhood of the diagonal of the state space $\left(s_{k}, s_{k}\right)$. In particular, for the topology of a homogeneous network, based on the assumption of the mean field, we can assume that the nodes are grouped on equal terms. As a result, the state of each node falls on the same square of the node to the state space $\left(s_{k}, s_{k}\right)$. So the equation can be expressed as

$$
p_{i j} \approx\left\{\begin{array}{l}
1, \text { when } i=j=k \\
0, \text { others }
\end{array}, k=1,2, \ldots, L\right.
$$

On the relationship between topology structure and the overall behavior of the network, we get the conclusion: the higher the degree of topology heterogeneity is, the greater the complexity of the flow fluctuation becomes. And thus the complexity of the overall behavior of the network is more conspicuous.

\section{References}

[1]. Henryk Fuks, Anna T Lawinczak. Performance of Data Networks with Random Links. Mathematics Computers in Simulation, 1999, 51 (1-2): 101-117.

[2]. T Ohira, Sawalari R. Phase Transition in a Computer Network Traffic Model. Phys. Rev. R, 1998, 58: 193-195

[3]. Dorogovtsev N., J.F.F. Mendes, A.N. Samukhin. Structure of Growing Networks with Preferential Linking. Phys. Rev. Lett., 2000, 85: 4633-4636.

[4]. T Nishikawa, Motter A E, Ying-Cheng Lai, et al. Heterogeneity in Oscillator Networks: Are Smaller worlds Easier to Synchronize? Phys. Rev. Lett, 2003, 91(1):014101.

[5]. R Albert, Barabasi A L. Statistical Mechanics of Complex Networks. Reviews of Modern Physics, 2002, (74): 78-94.

[6]. M DI Bernardo, Garofalo F, Manfredi S, et al. Load Distribution in Small World Networks. Proceeding of Physics and Control, 2005. 